Rodríguez Galán, M. y Gómez-Landero Rodríguez, L.A. (2017) Variables de rendimiento y penalizaciones técnicas del salto zancada / Performance Variables and Technical Penalties of the Split Leap. Revista Internacional de Medicina y Ciencias de la Actividad Física y el Deporte vol. 18 (72) pp. 605-619 Http://cdeporte.rediris.es/revista/revista72/artvariables966.htm

DOI: http://doi.org/10.15366/rimcafd2018.72.001

\title{
ORIGINAL
}

\section{VARIABLES DE RENDIMIENTO Y PENALIZACIONES TÉCNICAS DEL SALTO ZANCADA}

\section{PERFORMANCE VARIABLES AND TECHNICAL PENALTIES OF THE SPLIT LEAP}

\author{
Rodríguez Galán, M. ${ }^{1}$ y Gómez-Landero Rodríguez, L.A. ${ }^{2}$ \\ ${ }^{1}$ Graduada en Ciencias del Deporte INEF (UPM), Máster en Rendimiento Físico y Deportivo \\ (UPO). Departamento Deporte e Informática. Universidad Pablo Olavide. Sevilla (España) \\ mnica.rg@hotmail.com \\ 2 Profesor Asociado Doctor. Departamento Deporte e Informática. Universidad Pablo Olavide. \\ Sevilla (España) lagomrod@upo.es
}

AGRADECIMIENTOS: Los autores desean mostrar su agradecimiento a las gimnastas participantes en este estudio por su esfuerzo entusiasta e implicación durante la ejecución de la habilidad de salto analizada.

Código UNESCO / UNESCO Code: 2406.04 Biomecánica / Biomechanics. Clasificación del Consejo de Europa / Council of Europe Classification: 3. Biomecánica del deporte / Biomechanics of sport

Recibido 6 de diciembre de 2016 Received December 6, 2016 Aceptado 15 de enero de 2017 Accepted January 15, 2017

\section{RESUMEN}

Se analizó la relación entre las penalizaciones técnicas obtenidas en la ejecución de un elemento gimnástico aislado con el nivel competitivo de las gimnastas, sus características antropométricas y las variables temporales y angulares más importantes analizadas en dicha habilidad. Fueron seleccionadas 29 gimnastas $(17,1 \pm 3,1$ años) procedentes de distintos niveles competitivos. Tres entrenadores evaluaron la ejecución mediante una adaptación del Código de Puntuación. Se observaron asociaciones significativas directas e indirectas $(p$ $<0,05$ ): bajo índice de adiposidad ( $r h o=0,643$ ), tiempo total de batida ( $r$ ho $=$ 0,619 ), tiempo total de vuelo (rho $=-0,596$ ), máxima amplitud articular de caderas $($ rho $=-0,902)$ y máxima extensión de los tobillos (rho $=-0,738)$. La valoración de las penalizaciones así como el uso del nivel competitivo y del Salto Zancada como test específico han resultado ser herramientas útiles para la valoración del rendimiento. 
PALABRAS CLAVE: gimnasia rítmica, fotogrametría, rendimiento

\begin{abstract}
The aim of this study was to analyze the relationship between the following aspects: the technical penalties obtained in the execution of an isolated gymnastics element, the competitive level of the gymnasts, their anthropometric characteristics, and the most important temporal and angular variables analyzed in this skill. Twenty nine gymnasts (aged $17.1 \pm 3.1$ years) from different competition levels were selected. Three coaches evaluated the execution through an adaptation of the Code of Points. Significant direct and indirect associations were observed $(p<0.05)$ : low adiposity index (rho = 0.643), take-off total time (rho $=0.619$ ), flight total time (rho $=-0.596$ ), maximum range of motion of the hips ( $r$ o $=-0.902$ ), and maximum extension of ankles (rho $=-0.738$ ). The assessment of penalties as well as the use of competitive level and Split Leap as specific tests, have demonstrated to be useful tools for evaluating performance.
\end{abstract}

KEY WORDS: rhythmic gymnastics, photogrammetry, performance

\title{
INTRODUCCIÓN
}

El rendimiento en Gimnasia Rítmica (GR) viene definido por las notas o puntuaciones que otorgan los jueces durante el desarrollo del ejercicio en una competición, siguiendo la normativa que establece la Federación Internacional de Gimnasia (FIG) a través del Código de Puntuación (CoP). Es importante destacar que existen diferentes adaptaciones del CoP en la mayoría de países, con el fin de abarcar todos los niveles de competición en los cuales se desarrolla la práctica de la GR. Los jueces no evalúan de la misma manera a una gimnasta principiante que a una de élite, sino que se utilizan distintos criterios a la hora de evaluar gimnastas en los diferentes niveles de competición.

El CoP evalúa dos aspectos generales en la composición del ejercicio de GR: la ejecución técnica (E) y el valor de dificultad del ejercicio (D). Sin embargo, está diseñado para evaluar ejercicios enteros y no elementos aislados. Para analizar y evaluar elementos gimnásticos aislados, numerosos autores han desarrollado estudios biomecánicos en los cuales se evalúa la ejecución técnica en habilidades gimnásticas con el fin de detectar y corregir errores técnicos y, con ello, mejorar el rendimiento deportivo (Cicchella, 2009; Rodríguez, Villacieros y Ferro, 2013; Potop y Timnea, 2012; Se-Mi, Ji-Seon, y Tae-Sam, 2011; Tae-Sam, 2011; Byung-Hoon, Kae-san y Hee-Kyo, 2002). La mayoría de estos autores emplean algunas técnicas de la biomecánica deportiva, como la Fotogrametría (Aragón et al., 2010; Grande, Bautista y Hontoria, 2008; Cicchella, 2009; Mkaouer, Amara, y Tabka, 2012; Rodríguez et al., 2013) y el análisis de video en alta velocidad (Rodríguez et al., 2013; Mkaouer et al., 2014), para analizar patrones de movimientos y cuantificar variables cinemáticas (tiempos, ángulos, velocidad, etc.). 
Además de evaluar la ejecución técnica, otros tipos de variables (antropométricas, condicionales...) que han sido medidas en la literatura científica resultan ser decisivas para el rendimiento de las gimnastas. Muchos autores establecen la importancia de un bajo componente graso para la mejor ejecución de habilidades gimnásticas (Claessens et al., 1991; Claessens, Lefevre, Beunen, y Malina 1999; Douda, Toubekis, Avloniti, y Tokmakidis, 2008; Romero, Palomino y González, 2011; Miletic, Katic, y Males, 2004; Miletic y Kostic, 2006; Di Cagno et al., 2008, 2009; Hume, Hopkins, Robinson, Robinson y Hollings, 1993) así como la relevancia de las cualidades físicas de flexibilidad, fuerza explosiva y potencia para la mejor ejecución de habilidades de salto (Douda et al., 2008; Romero et al., 2011; Miletic et al., 2004; Miletic y Kostic, 2006; Hume et al., 1993). La fuerza explosiva y la potencia se manifiestan tanto en la ejecución de test de salto generales (CMJ, SJ, o Hopping test) como en la ejecución de test de salto específicos de GR, estableciéndose una relación entre ambos tipos de test (Di Cagno et al., 2008, 2009; Romero et al., 2011). La flexibilidad solo se manifiesta en test de salto específicos de GR, debido al elevado requerimiento de amplitud de movimiento que exige la ejecución de este tipo de habilidades (Di Cagno et al., 2008, 2009; Romero et al., 2011).

De todos los saltos específicos de GR, el Salto Zancada (SZ) es el más ampliamente utilizado en la bibliografía científica (Di Cagno et al., 2008, 2009; Miletic et al., 2004; Miletic y Kostic, 2006; Grande et al., 2008; Dyhre-Poulsen, 1987; Cicchella, 2009; Mkaouer et al., 2012; Rodríguez et al., 2013) debido a las siguientes razones principales: es uno de los saltos más característicos de este deporte, es un elemento sencillo que aporta multitud de información útil para el entrenador (Cicchella, 2009; Grande et al., 2008; Rodríguez et al., 2013), y, por último, es una habilidad que se enseña desde la iniciación deportiva y que continúa empleándose en el resto de niveles de competición.

La evaluación cualitativa de los jueces es el principal medio de valorar el rendimiento de la GR en competición. El sistema de puntuación está orientado a evaluar un ejercicio entero en lugar de elementos aislados (FIG, 2013). Considerando lo anterior, y dada la evidencia de la influencia de ciertos tipos de variables en la ejecución de habilidades gimnásticas aisladas, podríamos establecer la hipótesis de que la evaluación cualitativa del SZ podría verse influida por la existencia de una posible correlación entre variables antropométricas y cinemáticas. Esto podría proporcionar suficiente información para indicar o estimar el nivel competitivo de la gimnasta. Por este motivo, los objetivos de este estudio son los siguientes: 1) proponer una forma sencilla de evaluar la ejecución técnica en un elemento aislado (SZ) mediante una adaptación del CoP, y 2) analizar con ello las posibles relaciones entre las penalizaciones técnicas observadas, las características antropométricas y el nivel competitivo de la gimnasta, así como las variables cinemáticas más importantes analizadas en el SZ.

\section{MATERIAL Y MÉTODOS}




\section{Participantes}

Un total de 29 mujeres, gimnastas de GR (edad = 17,1 \pm 3,1 años, experiencia deportiva $=9,3 \pm 3,5$ años), fueron seleccionadas para este estudio a través de un proceso de selección llevado a cabo siguiendo el consejo de expertos (entrenadores de GR). Los criterios de selección fueron los siguientes: en primer lugar, las gimnastas debían saber ejecutar la habilidad objeto de análisis, la cual debía ser una habilidad ya adquirida y no un elemento nuevo a aprender; en segundo lugar, con objeto de asegurar un adecuado nivel de aprendizaje de la habilidad así como consistencia y seguridad en su ejecución, las gimnastas debían de tener al menos cinco años de experiencia en GR, tiempo considerado suficiente por algunos autores para aprender y automatizar habilidades motrices básicas (Zisi Giannitsopoulou, Vassiliadou, Pollatou, y Kiomourtzoglou, 2009; Miletic et al., 2004, Douda et al., 2008); en tercer lugar, la muestra debía contar con gimnastas de distintos niveles competitivos (provincial: $n=10$, regional: $n=5$, nacional: $n=12$, internacional: $n=2$ ) (Douda et al., 2008; Romero et al., 2011). Todas las gimnastas aceptaron participar en el estudio de manera voluntaria mediante un consentimiento informado, firmado por las mismas o bien por sus tutores legales. Este consentimiento cumplía con la normativa ética para investigación en humanos, según los principios de la Declaración de Helsinki (Asociación Médica Mundial, W.M.A., 2008).

\section{Procedimientos}

El desarrollo de este estudio fue llevado a cabo en tres etapas:

\subsection{Medición y registro de las variables antropométricas}

Los datos antropométricos fueron recogidos de cada gimnasta empleando protocolos estandarizados (Marfell-Jones, Olds, Stewart, y Carter, 2006; Esparza, 1993). Las variables antropométricas incluidas en este estudio fueron: peso, estatura, índice de masa corporal (IMC), cuatro pliegues cutáneos (tríceps, subescapular, abdominal, supra-ilíaco), y sumatorio de los cuatro pliegues. Para la medición de la estatura se utilizó un tallímetro de pared. El peso fue medido utilizando una báscula digital con precisión de 0,1 kg (Laica SpA, Vicenza, Italy). Para la medición de los pliegues cutáneos se utilizó un plicómetro (Slim Guide (B), Creative Health Products, Michigan, USA) con precisión de 0,5 mm. Todas las mediciones de los pliegues se realizaron por triplicado por el mismo observador, en el lado derecho del cuerpo, con los participantes en posición de pie, utilizando un rotulador dermográfico, con lectura a los cuatro segundos en cada medida y con un periodo de pausa entre mediciones de entre 1-2 minutos.

\subsection{Elección de la habilidad gimnástica a evaluar}

Se escogió un elemento que representa y compone una de las habilidades propias y características de la GR y que, por tanto, forma parte de su estructura motora: un salto. En concreto se escogió el SZ. Según el CoP (FIG, 2013), se trata de un salto en el que se muestra la figura del espagat. El motivo de la elección de esta habilidad se basa en los siguientes criterios: en primer lugar, se 
trata de una habilidad de la GR muy básica y simple que toda gimnasta debe conocer puesto que se enseña y aprende desde la iniciación deportiva; en segundo lugar, existe una progresión hacia habilidades de salto de mayor dificultad y valor técnico (FIG, 2013).

\subsection{Medición y registro de las variables cinemáticas}

En este estudio se ha seguido la metodología propuesta por Rodríguez et al. (2013) para el análisis cinemático de la técnica. En primer lugar, las gimnastas fueron filmadas ejecutando el SZ a una frecuencia de 240 fotogramas por segundo utilizando una cámara de vídeo de alta velocidad (Casio Exilim EXZR200, Tokyo, Japan). Después, los vídeos filmados fueron analizados mediante el programa informático Kinovea 0.8.15. (Kinovea.org, France). Dos tipos de variables cinemáticas fueron cuantificadas para el análisis de la ejecución técnica del SZ: variables Temporales (tiempo total de las fases del movimiento: batida, vuelo, máxima amplitud articular, recepción y ejecución global del salto) y variables Angulares (ángulos de máxima amplitud articular de las articulaciones de los miembros inferiores durante la fase de vuelo: extensión del tobillo de la pierna de delante, extensión del tobillo de la pierna de atrás, flexión de la cadera de la pierna de delante, extensión de la cadera de la pierna de atrás, y máxima amplitud articular entre las dos caderas).

\subsection{Registro de las variables criterio}

En este estudio se utilizaron dos tipos de variables relacionadas con el rendimiento en GR: Nivel Competitivo y Nota de Penalización en la ejecución del SZ.

Por un lado, para representar el nivel de rendimiento deportivo de las gimnastas participantes en este estudio y diferenciarlas, se ha establecido una categorización del Nivel Competitivo, siguiendo el consejo y los conocimientos de varios expertos (entrenadores de GR) y gracias a la revisión de estudios previos (Gómez-Landero, López y Vernetta, 2013; Zisi et al., 2009). Esta categorización fue definida en seis niveles de competición: Nivel 1: Competición Provincial Básica (iniciación deportiva), Nivel 2: Competición Provincial Especializada (promoción deportiva), Nivel 3: Competición Nacional-Autonómica (resultados en clasificación medios-bajos), Nivel 4: Competición NacionalAutonómica (resultados en clasificación medios-altos), Nivel 5: Competición Nacional-Autonómica (medallistas nacionales) y Nivel 6: Competición Internacional o Miembros de la Selección Nacional. Estos seis niveles han sido establecidos en función del tipo de competición en el que participen las gimnastas y en función de los resultados clasificatorios en competiciones oficiales (quedar entre los primeros puestos y/o ser medallistas, quedar por la mitad, por debajo de la mitad, o en los últimos puestos).

Además, por otro lado, se realizó una adaptación del CoP para evaluar de forma sencilla el rendimiento en la ejecución de elementos de GR aislados (Miletic et al., 2004; Miletic y Kostic, 2006; Delas, Miletic, y Miletic, 2008). En el sistema de puntuación del CoP (FIG, 2013), los jueces de Ejecución van 
restando las penalizaciones observadas sobre una nota inicial de 10 puntos. En el presente trabajo se han sumado las penalizaciones o errores técnicos observados, obteniendo una Nota de Penalización. Esta Nota de Penalización se basa en una escala de valoración de 0 a 5 , y ha sido constituida en relación a los siguientes aspectos cualitativos: amplitud, forma y fijación de la dificultad (extensión de tobillos y rodillas, fijación de la posición, y mínima amplitud articular requerida) (FIG, 2013). La escala se define de la siguiente manera: 0 = ningún fallo (perfecta ejecución de la dificultad, exenta de errores técnicos); $1=1$ fallo (ejemplo: falta de amplitud); 2 = 2 fallos (ejemplo: falta de amplitud y fijación); 3 = 3 fallos (ejemplo: falta de amplitud, fijación, no extensión de rodillas); $4=4$ fallos (ejemplo: falta de amplitud, fijación, no extensión de tobillos, ...); $5=5$ fallos (dificultad muy mal ejecutada); > 5 fallos = No se considera la dificultad como aprendida. Mediante esta escala se pretende categorizar la calidad técnica de ejecución de cada una de las gimnastas como un medio sencillo de medir o evaluar el rendimiento deportivo. Para ello, tres entrenadores de GR (las cuales poseían el título de entrenador nacional de GR junto con una alta y demostrable experiencia en este deporte) accedieron voluntariamente a valorar la ejecución técnica del SZ, visualizando los vídeos filmados previamente de cada una de las gimnastas. Estos entrenadores eran capaces de distinguir y evaluar errores técnicos en dificultades corporales o habilidades de GR.

\section{Análisis Estadístico}

El análisis estadístico fue llevado a cabo mediante el programa SPSS versión 22.0.0.0 (IBM Corp., Armonk, NY, USA). El análisis de la distribución de los datos se realizó mediante el test de normalidad de Shapiro-Wilk. Se efectuó un análisis de la fiabilidad en aquellas variables que fueron evaluadas en más de una ocasión. En el caso de las medidas de los pliegues, se ha efectuado un análisis de la fiabilidad relativa, por medio del coeficiente de correlación intraclase $(\mathrm{CCl})$, y de la fiabilidad absoluta a través del coeficiente de variación (CV). El CCI se calculó teniendo en cuenta la diferencia de medidas y con un intervalo de confianza del 95\%, a través de un análisis de la varianza (ANOVA) de un factor para medidas repetidas. Para el análisis de la fiabilidad entre las puntuaciones otorgadas en el SZ, se calculó el coeficiente de concordancia W de Kendall entre los tres observadores. Se llevó a cabo un análisis descriptivo de los grupos de variables del estudio. Finalmente, se halló el coeficiente de correlación de Spearman (Rho) para analizar las asociaciones entre variables. El nivel de significación fue establecido en un 0,05 para todos los análisis estadísticos. Siguiendo a Hopkins (2006), la magnitud del coeficiente de correlación se consideró trivial $(0-0,1)$, pequeña $(0,1-0,3)$, moderada $(0,3-0,5)$, grande $(0,5-0,7)$, muy grande $(0,7-0,9)$ y casi perfecta $(0,9-1)$.

\section{RESULTADOS}

Respecto a los resultados del análisis de fiabilidad de medidas repetidas (Tabla 1), los valores de CCl observados en los cuatro pliegues cutáneos fueron 
superiores a 0,9. Estos datos sugieren que las medidas realizadas son fiables y que poseen gran validez interna. Respecto a la fiabilidad absoluta de estas medidas, el CV que presentan las mediciones de los cuatro pliegues cutáneos son inferiores al 5\% máximo aconsejado por el GREC (Esparza, 1993).

Tabla 1. CCl y CV de las mediciones de los pliegues cutáneos.

\begin{tabular}{ccccc}
\hline $\begin{array}{c}\text { Pliegue } \\
\text { (n=29) }\end{array}$ & CV (\%) & CCI & $\begin{array}{c}\text { Límite } \\
\text { Inferior }\end{array}$ & $\begin{array}{c}\text { Límite } \\
\text { superior }\end{array}$ \\
\hline Tríceps & 1,94 & 0,998 & 0,996 & 0,999 \\
Subescapular & 2,22 & 0,997 & 0,995 & 0,999 \\
Abdominal & 2,67 & 0,996 & 0,993 & 0,998 \\
Supra-ilíaco & 2,28 & 0,998 & 0,996 & 0,999 \\
\hline
\end{tabular}

$\mathrm{CCl}$, coeficiente de correlación intraclase; CV, coeficiente de variación; IC, intervalo de confianza.

En cuanto a los resultados de concordancia entre las Notas de Penalización otorgadas por los tres observadores (Tabla 2), se observa un elevado valor del coeficiente $\mathrm{W}$ de Kendall (próximo a 1), junto con un alto nivel de significación $(p<0,01)$. Por lo tanto, la concordancia entre los tres observadores es significativa y muestra una gran fiabilidad (Siegel y Castellan, 1995).

Tabla 2. Coeficiente de concordancia W de Kendall entre los tres observadores.

\begin{tabular}{ccc}
\hline $\begin{array}{c}\text { Valoración cualitativa de la ejecución } \\
\text { técnica (Escala 0-5) }\end{array}$ & W de Kendall & Chi-cuadrado \\
\hline $\begin{array}{c}\text { Nota de Penalización en el SZ } \\
(n=29)\end{array}$ & $0,798^{* *}$ & 67,054 \\
\hline${ }^{* *} p<0,01$ & &
\end{tabular}

Se obtuvieron asociaciones significativas $(p<0,01)$ entre las variables antropométricas y cinemáticas analizadas con la Nota de Penalización en el SZ, así como con el Nivel Competitivo de las gimnastas (Tabla 3). Todas las variables antropométricas fueron asociadas en gran parte con la Nota de Penalización en el SZ, excepto la estatura. Los pliegues tríceps, abdominal y supra-ilíaco, así como el IMC y la suma de los cuatro pliegues cutáneos se asociaron de forma moderada e indirecta con el Nivel Competitivo $(p<0,05)$. En cuanto a las variables cinemáticas, se observaron asociaciones grandes y muy grandes (rho $>0,7)$ entre la Nota de Penalización, el Nivel Competitivo y todas las variables angulares. La única variable que ha demostrado tener mayor asociación con las variables criterio es el ángulo de máxima amplitud articular entre las dos caderas (Figura 1). El tiempo total de batida y el tiempo total de vuelo fueron significativamente correlacionados $(p<0,01)$ con la Nota de Penalización en el SZ y con el Nivel Competitivo de la gimnasta. El tiempo total de máxima amplitud articular no ha mostrado correlación significativa con ninguna de las variables de criterio (Tabla 3).

Tabla 3. Correlaciones significativas entre las variables antropométricas y cinemáticas con las variables criterio. 


\begin{tabular}{|c|c|c|}
\hline $\begin{array}{l}\text { Variables analizadas } \\
\qquad(\mathrm{n}=29)\end{array}$ & $\begin{array}{c}\text { Nota de } \\
\text { Penalización en el } \\
\text { SZ (0-5) }\end{array}$ & $\begin{array}{c}\text { Nivel } \\
\text { Competitivo } \\
(1-6)\end{array}$ \\
\hline \multicolumn{3}{|l|}{ Estatura $(\mathrm{cm})$} \\
\hline Peso $(\mathrm{kg})$ & $0,580^{* *}$ & \\
\hline Pliegue Tríceps (mm) & $0,590^{* *}$ & $-0,387^{*}$ \\
\hline Pliegue Subescapular (mm) & $0,592^{* *}$ & \\
\hline Pliegue Abdominal (mm) & $0,645^{\star *}$ & $-0,435^{*}$ \\
\hline Pliegue Supra-ilíaco (mm) & $0,627^{\star *}$ & $-0,438^{*}$ \\
\hline IMC $\left(\mathrm{kg} / \mathrm{m}^{2}\right)$ & $0,628^{* *}$ & $-0,433^{*}$ \\
\hline Sumatorio de los pliegues $(\mathrm{mm})$ & $0,643^{* *}$ & $-0,405^{*}$ \\
\hline Tiempo total Batida (s) & $0,619^{* *}$ & $-0,577^{* *}$ \\
\hline Tiempo total Vuelo (s) & $-0,596^{* *}$ & $0,636^{* *}$ \\
\hline \multicolumn{3}{|l|}{ Tiempo total Máxima Amplitud Articular (s) } \\
\hline Extensión Tobillo adelante $(\stackrel{\circ}{\circ})$ & $-0,737^{* *}$ & $0,782^{* *}$ \\
\hline Extensión Tobillo atrás $(\stackrel{\circ}{\circ})$ & $-0,738^{* *}$ & $0,582^{* *}$ \\
\hline Flexión Cadera adelante $(\stackrel{\circ}{*})$ & $-0,791^{* *}$ & $0,614^{* *}$ \\
\hline Extensión Cadera atrás $(\stackrel{\circ}{*})$ & $-0,811^{* *}$ & $0,821^{* *}$ \\
\hline Máxima amplitud articular (ํ) & $-0,902^{* *}$ & $0,823^{* *}$ \\
\hline
\end{tabular}

${ }^{* *} p<0,01 ;{ }^{*} p<0,05$

\section{DISCUSIÓN}

En este estudio ha sido analizada la relación entre las penalizaciones técnicas observadas en el SZ (obtenidas mediante una adaptación del CoP de la FIG), el nivel competitivo de las gimnastas, sus características antropométricas, y las variables angulares y temporales más importantes del salto. De entre los resultados más representativos obtenidos, destacan tres aspectos como los más relevantes: 1) un gran tejido adiposo, 2) un bajo ángulo de amplitud de caderas y 3 ) un elevado tiempo de la fase de batida, todos ellos se asocian con penalizaciones más altas y con un menor nivel competitivo.

Los principales resultados del presente trabajo mostraron que las variables que evalúan indirectamente la adiposidad (peso corporal, IMC, pliegues cutáneos y la suma de los mismos) se han asociado con la ejecución del SZ. Un mayor peso corporal se ha relacionado con una mayor penalización, y por tanto con una peor ejecución (Tabla 3). Nuestros resultados coinciden con lo que afirman diversos estudios: el peso corporal es un factor fundamental a tener en cuenta de cara al óptimo rendimiento físico en GR (Camargo et al., 2014; Vernetta, Fernández, López-Bedoya, Gómez-Landero y Oña, 2011). Además de suponer una desventaja para el rendimiento, el exceso de peso en gimnastas de GR favorece la aparición de lesiones deportivas fundamentalmente a la hora de ejecutar habilidades o dificultades corporales de salto dado que resultaría lesivo para las articulaciones de los miembros inferiores (tobillos y rodillas) (Zetaruk, Violan, Zurakowski, Mitchell y Micheli, 2006). Estos resultados se ven reforzados por la asociación observada entre el IMC y la ejecución del SZ, por lo que una mayor masa corporal parece también influenciar un bajo rendimiento. Estos resultados apoyan lo expuesto por Douda et al (2008) quienes afirman que tener un bajo IMC parece ser una ventaja cuando se realizan 
habilidades que requieren movimientos complejos. Estos autores también afirman que en gimnastas de élite el $8,5 \%$ de la varianza del rendimiento es explicada por el IMC. Además, nuestros resultados apoyan los obtenidos por otra investigación en donde los gimnastas que poseían mayores valores en las medidas antropométricas (estatura, peso...) fueron los que peores puntaciones obtuvieron en competición (Claessens et al., 1991).

Muchos de los trabajos revisados analizan el componente adiposo distinguiéndolo del peso total o IMC, y destacando la relación entre una mayor cantidad de tejido graso con un peor rendimiento en habilidades gimnásticas; estos estudios concuerdan con las relaciones observadas entre los cuatro pliegues cutáneos evaluados y las notas de penalización. Una mayor cantidad de tejido graso subcutáneo se ha relacionado con un peor rendimiento en el SZ. Estos resultados son similares a los expuestos previamente por diversos autores (Claessens et al., 1991, 1999; Romero et al., 2011).

De la misma manera, el sumatorio de los cuatro pliegues también ha demostrado estar relacionado con una mejor ejecución del SZ (Tabla 3). Éste es un índice indirecto de la adiposidad el cual supone una valoración más objetiva en la estimación y control del índice de adiposidad que el IMC (Esparza, 1993). En este estudio, un bajo nivel de grasa subcutánea ha sido relacionado con el mejor rendimiento en el SZ, así como con el nivel competitivo más elevado. Nuestros resultados coinciden con aquellos trabajos que verifican que un bajo porcentaje de grasa en gimnastas de GR es determinante del rendimiento deportivo, siendo éste definido como la puntuación o clasificación obtenida en competición (Miletic et al., 2004; Claessens et al., 1991, 1999; Douda et al., 2008). Por otra parte, todo movimiento o habilidad de GR requiere ser ejecutada eficientemente, para lo cual un bajo volumen de tejido adiposo es fundamental (Miletic et al., 2004; Miletic and Kostic, 2006). Además, este estudio confirma lo que varios autores han indicado en estudios previos: las gimnastas de GR están caracterizadas por poseer un bajo porcentaje de grasa en comparación no sólo con mujeres sedentarias de la misma edad, sino en comparación con otras poblaciones de deportistas (Vernetta et al., 2011; Miletic et al., 2004; Menezes and Filho, 2006; Camargo et al., 2014).

Por otra parte, nuestros resultados también han demostrado que el índice de adiposidad se ha asociado con el Nivel Competitivo, por lo que un mayor nivel competitivo está vinculado con un menor nivel de adiposidad. Estos resultados coinciden con estudios que analizan el perfil antropométrico de las gimnastas de GR destacando el bajo porcentaje de grasa como variable fundamental (Vernetta et al., 2011; Miletic et al., 2004; Menezes and Filho, 2006; Camargo et al., 2014).

En cuanto a las variables angulares evaluadas, otro de los resultados más importantes de este estudio fue la importancia de la extensión de las articulaciones de los tobillos y la amplitud de las caderas en el SZ. Los ángulos de máxima extensión de los tobillos se han asociado con penalizaciones más bajas y por tanto con mejores niveles de ejecución, lo cual indica y demuestra la importancia que tiene en los deportes gimnásticos, en especial en la GR, el hecho de mantener los pies o "puntas estiradas" afín de lograr la estética requerida en los movimientos (FIG, 2013) y, por consiguiente, una mejor calidad 
y valoración de los mismos. El ángulo de máxima amplitud articular entre ambas caderas presenta una asociación elevada con una mejor ejecución del salto (Figura 1A). Este dato representa: 1) la importancia de la máxima amplitud articular posible en la ejecución de habilidades de salto específicas de GR, y 2), por consiguiente, la relevancia de la flexibilidad en su manifestación activabalística en este tipo de dificultades corporales. Estos resultados coinciden con varios autores y trabajos (Mendizábal, 2001; Grande et al., 2008; Rodríguez et al., 2013; Romero et al., 2011; Miletic et al., 2004; Miletic y Kostic, 2006; Volpi da Silva, Lopez, Grillo, Moya, y Matsushigue, 2008).

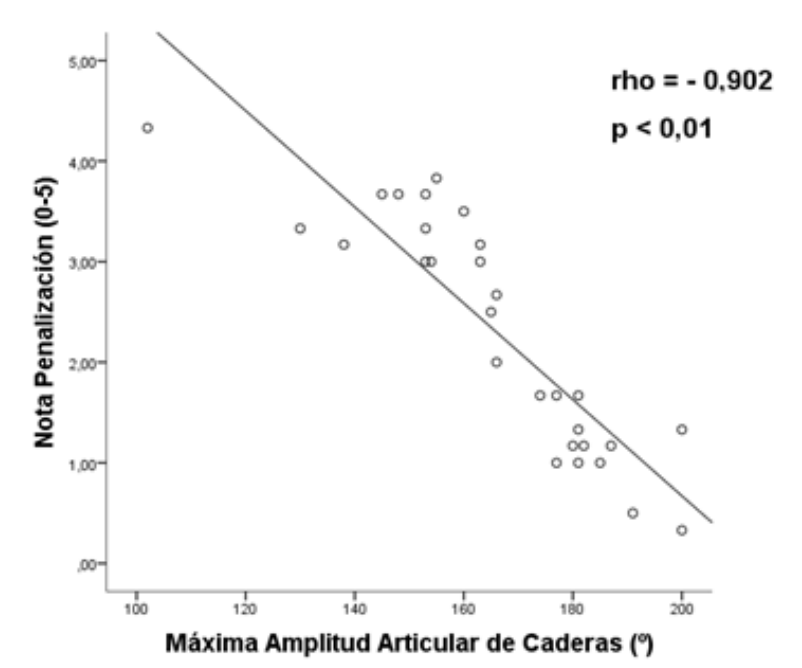

A

Figura 1.- Correlaciones entre el ángulo de máxima amplitud articular y A) Nota de Penalización en el SZ, y B) Nivel Competitivo. Datos para todos los sujetos $(n=29)$.

Por otro lado, este estudio destaca la importancia del tiempo total de las fases de batida y de vuelo en la ejecución del SZ. Estas variables cinemáticas temporales han sido asociadas con una mejor ejecución de esta dificultad corporal. Según Hay (1993), en la fase de batida se requiere fuerza explosiva en los miembros inferiores para generar la acción de impulso (la cual debe realizarse con gran velocidad y coordinación entre las articulaciones involucradas). En este trabajo, se ha observado que cuanto más tiempo emplee una gimnasta en ejecutar la fase de batida, peor nivel de rendimiento obtendrá en la ejecución del salto (Figura 2). Por otro lado, la duración de la fase de vuelo depende de la calidad de la batida. Cuanto mejor sea la batida, mayores posibilidades tendrá la gimnasta para realizar acciones corporales específicas y mayor será el grado de dificultad que podrá lograr con el salto (Hay, 1993). Nuestros resultados se relacionan con las cuantificaciones cinemáticas respecto al tiempo de duración de las fases de batida y de vuelo realizadas en un SZ de GR que proponen Grande et al. (2008). También, nuestros resultados coinciden con lo expuesto por Rodríguez et al. (2013), quienes analizaron la secuencia temporal de este mismo salto comparando la ejecución de distintas gimnastas mostrando que el tiempo de batida y de vuelo resultaban determinantes para su correcta ejecución, y a su vez que a las gimnastas podían distinguirse por su nivel técnico. Por último, dado que el CoP establece una serie de requisitos para 
la correcta ejecución de los saltos de la GR ("forma definida y fijada" y "altura suficiente para mostrar la forma correspondiente") se recalca la importancia de la duración de la fase de vuelo, con lo que nuestros resultados obtenidos sobre esta fase están de acuerdo con lo exigido por el propio CoP (FIG, 2013). Así, cuanto mayor sea el tiempo que permanece la gimnasta en la fase de vuelo, más tiempo tendrá para mostrar y mantener o fijar la forma del salto (en esta caso, zancada), por lo que la calidad técnica de ejecución será mayor y el salto será mejor valorado.

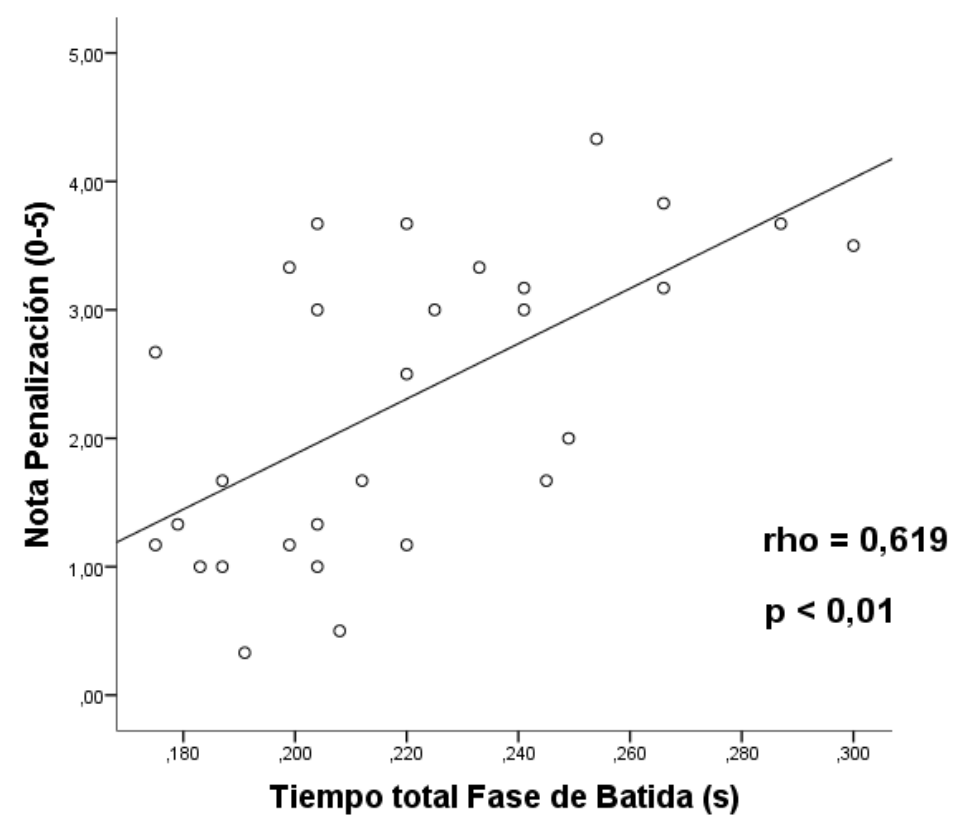

Figura 2.- Tiempo total de Batida en relación con la Nota de Penalización en el SZ. Datos para todos los sujetos $(n=29)$.

Finalmente, el último de los resultados más importantes obtenidos en este estudio resalta la importancia del nivel competitivo como variable criterio, lo que ha demostrado su relación con la mejor ejecución técnica del SZ. También presenta numerosas asociaciones con las variables analizadas, especialmente con la máxima amplitud articular de las caderas (Figura 1B). De manera que el rendimiento observado en la dificultad corporal evaluada (SZ) se ha asociado con un cierto nivel competitivo. Nuestros resultados son similares a los obtenidos por otra investigación (López y Vernetta, 1997).

\section{CONCLUSIONES}

En este estudio se ha corroborado el perfil antropométrico de las gimnastas de GR (bajo peso corporal, bajo IMC, bajo nivel de grasa subcutánea) y, además, los valores más bajos de tejido adiposo han mostrado los mejores resultados en el SZ. Por otro lado, el tiempo total de batida, el tiempo total de vuelo y el máximo rango de movimiento ( $\mathrm{y}$, en consecuencia, la cualidad física de flexibilidad, particularmente en su manifestación activa-balística) resultan ser determinantes para la ejecución correcta del SZ. Además, el uso de filmaciones en alta velocidad (a $240 \mathrm{fps}$ ) nos ha permitido visualizar aquellos detalles técnicos que el entrenador o el propio deportista no hubiesen apreciado mediante cámaras de vídeo de menor frecuencia o a simple vista. Así, en este trabajo, se 
ha podido observar la importancia que tiene mantener los pies o las "puntas estiradas" en la ejecución del SZ. Todas estas variables que se han asociado con la ejecución del SZ nos hacen deducir algunos de los requerimientos físicos que demanda la práctica de GR para la ejecución de las dificultades corporales de salto: velocidad, fuerza relativa, fuerza explosivo-elástica y, sobre todo, flexibilidad activa-balística. Por lo tanto, mediante el conocimiento acerca de cuáles son las variables que más influyen en la ejecución de las dificultades corporales de GR, se podría incidir sobre la mejora de dichos aspectos durante los procesos de entrenamiento y lograr así incrementar el rendimiento de las gimnastas: conseguir la máxima nota o clasificación en competición.

Por otro lado, se han establecido relaciones importantes entre el rendimiento observado en el SZ y el nivel competitivo de las gimnastas. Un determinado nivel de rendimiento se ha asociado con un determinado nivel competitivo. Sin embargo, estos resultados se ven limitados en este trabajo dada la heterogeneidad de la muestra de gimnastas según sus niveles de competición. Por ello, se propone la realización de nuevos estudios con una muestra homogénea de este tipo a fin de obtener una información más relevante. Por último, el uso de pruebas de salto específicas como batería de test ofrece información útil y valiosa para la GR dado que se trata de una modalidad deportiva con gran demanda de habilidades de salto. Además, la evaluación cualitativa de la ejecución técnica mediante la suma de penalizaciones ha demostrado ser una herramienta útil para la evaluación del rendimiento y el nivel competitivo de las gimnastas, ya que proporciona información útil para el entrenador dada su objetividad.

\section{REFERENCIAS BIBLIOGRÁFICAS}

Aragón, J., Fernández, J., Gómez, R., Carrasco, A., Mora, J. and González, J.L. (2010) Kinematic analysis of throwing between rigth and left top member in wáter-polo. International Journal of Medicine and Science of Physical Activity and Sport, 10(39), 369-379.

Byung-Hoon, W., Kae-san, L. and Hee-Kyo, I. (2002) Analysis of Kinematic on Ring jump in the Rhythmic Sport Gymnastics. Korean Journal of Sport Biomechanics, 12(2), 65-76.

DOI: https://doi.org/10.5103/KJSB.2002.12.2.065

Camargo, C.T., Gomez-Campos, R.A., Cossio-Bolaños, M.A., Barbeta, V.J., Arruda, M. and Guerra-Junior, G. (2014) Growth and body composition in Brazilian female rhythmic gymnastics athletes. Journal of Sport Sciences, 32(19), 1790-1796.

DOI: https://doi.org/10.1080/02640414.2014.926381

Cicchella, A. (2009) Kinematics analysis of selected rhythmic gymnastics leaps. Journal of Human Sport and Exercise, 4(1), 40-47.

DOI: https://doi.org/10.4100/jhse.2009.41.05

Claessens, A.L., Lefevre, J., Beunen, G., Stijnen, V., Maes, H. and Veer, F.M. (1991) Gymnastics performance as related to anthropometric and somatotype characteristics in male gymnasts. Anthropology, 33, 243-247.

Claessens, A.L., Lefevre, J., Beunen, G. and Malina, R. (1999) The contribution of anthropometric characteristics to performance scores in elite female 
gymnasts. Journal of Sports Medicine and Physical Fitness, 39(4), 355360.

Delas, S., Miletic, A. and Miletic, D. (2008) The influence of motor factors on performing fundamental movement skills - The differences between boys and girls. Facta Universitatis, Series: Physical Education and Sport, 6(1), 31-39.

Di Cagno, A., Baldari, C., Battaglia, C., Brasili, P., Merni, F., Piazza, M., Toselli, S., Ventrella, A.R. and Guidetti, L. (2008) Leaping ability and body composition in rhythmic gymnasts for talent identification. Journal of Sports Medicine and Physical Fitness, 48(3), 341-346.

Di Cagno, A., Baldari, C., Battaglia, C., Monteiro, M.A., Pappalardo, A., Piazza, M. and Guidetti, L. (2009) Factors influencing performance of competitive and amateur rhythmic gymnastics-Gender differences. Journal of Science and Medicine in Sport, 12, 411-416.

DOI: https://doi.org/10.1016/j.jsams.2008.01.006

Douda, T., Toubekis, A., Avloniti, A. and Tokmakidis, S. (2008) Physiological and anthropometric determinants of Rhythmic gymnastics performance. International Journal of Sports Physiology and Performance, 3, 41-54.

DOI: https://doi.org/10.1123/ijspp.3.1.41

Dyhre-Poulsen, P. (1987) An analysis of split leaps and gymnastics kill by physiological recordings. European Journal of Applied Physiology and Occupational Physiology, 56(4), 390-397.

DOI: https://doi.org/10.1007/BF00417765

Esparza, F. (1993) Kinanthropometry Manual: Monographs FEMEDE. $1^{\text {st }}$ ed. Madrid, Spain: GREC FEMEDE.

FIG (2013) Code of Points 2013-2016. International Gymnastics Federation.

Gómez-Landero, L.A., López, J. and Vernetta, M. (2013) Active and passive flexibility evaluation in spanish trampolinists. International Journal of Medicine and Science of Physical Activity and Sport, 13(49), 5572.

Grande, I., Bautista, A. and Hontoria, M. (2008) Applied Biomechanics to the design of a tool for rhythmic gymnastics to evaluate jumps taking account the International Code of Points. Apunts: Physical Education and Sports, 3, 55-61.

Hay, J.G. (1993) The Biomechanics of Sports Techniques. 4th Ed. New Jersey, US: Prentice-Hall, Inc.

Hopkins, W.G. (2006) A scale of magnitudes for effect statistics. http://www.sportsci.org/resource/stats/effectmag.html. Accessed July 2014.

Hume, P., Hopkins, W., Robinson, D.M., Robinson, S.M. and Hollings, S. (1993) Predictors of attainment in rhythmic sportive gymnastics. Journal of Sports Medicine and Physical Fitness, 33(4), 367-377.

López, J. and Vernetta, M. (1997) Application of a basic gymnastic test for talent identification in artistic gymnastics in the generic phase of adaptation and initiation to physical and sporting activities. European Journal of Human Movement, 3, 67-87.

Marfell-Jones, M., Olds, T., Stewart, A. and Carter, L. (2006) International standards for anthropometric assessment. Potchefstroom, South Africa: ISAK. 
Mendizábal, S. (2001) Fundamentals of the Rhythmic Gymnastics: Myths and realities. Madrid, Spain: Gymnos.

Menezes, L.S. and Filho, J.F. (2006) Identification and comparison of dermatoglyphics, somatotype and basic physical aptitude characteristics of rhythmic gymnasts of different qualification levels. Fitness \& Performance Journal, 5(6).

Miletic, D., Katic, R. and Males, B. (2004) Some anthropologic factors of performance in rhythmic gymnastics novices. Collegium Antropologicum, 28(2), 727-737.

Miletic, D. and Kostic, R. (2006) Motor and morphological conditionality for performing arabesque and passe pivots. Facta Universitatis Series: Physical Education and Sport, 4(1), 17-25.

Mkaouer, B., Amara, S. and Tabka, Z. (2012) Split leap with and without ball performance factors in rhythmic gymnastics. Science of Gymnastics Journal, 4(2), 75.

Mkaouer, B., Jemni, M., Amara, S., Chaabène, H., Padulo, J. and Tabka, Z. (2014) Effect of Three Technical Arms Swings on The Elevation of the Center of Mass During a Standing Back Somersault. Journal of Human Kinetics, 40, 37-48.

DOI: https://doi.org/10.2478/hukin-2014-0005

Potop, V. and Timnea, O. (2012) Comparative biomechanical analysis of key elements in stretched salto backward yurchenko vault. Journal of Physical Education and Sport, 12(4), 521.

Rodríguez, M., Villacieros, J. and Ferro, A. (2013) Analysis of the coordination and the sequence of corporal movements in the Split Leap of rhythmic gymnastics. In: Rojas, F.J. and Gutiérrez, C. (Eds.) 34th Congress of the Iberian Society of Biomechanics and Biomaterials (p. 78) Granada, Spain: University of Granada.

Romero, B., Palomino, A. and González, J.J. (2011) The Rhythmic Gymnastics anthropometric profile. Apunts: Physical Education and Sports, 103, 48.

Se-Mi, S., Ji-Seon, R. and Tae-Sam, K. (2011) The Biomechanical Analysis of two and half rotation technic of Penche in Rhythmic Gymnastics. Korean Journal of Sport Biomechanics, 21(3), 269-279.

DOI: https://doi.org/10.5103/KJSB.2011.21.3.269

Siegel, S. and Castellan, N.J. (1995) Nonparametric statistics applied to behavioral sciences. México: Trillas.

Tae-sam, K. (2011) Variability of kinematical factors during consecutive 9 backward illusion motion at rhythmic gymnastics. The Korean Society of Sports Science, 20(3), 1679-1696.

Vernetta, M., Fernández, E., López-Bedoya, J., Gómez-Landero, A. and Oña, A. (2011) A study of the Morphological and Psychological Characteristics of the Andalusian Rhythmic Gymnastics Team. European Journal of Human Movement, 26, 77-92.

Zetaruk, M., Violan, M., Zurakowski, D., Mitchell, W. and Micheli, L. (2006) Recommendations for the training and injury prevention in elite rhythmic gymnasts. Apunts: Physical Education and Sports, 151, 100-106.

Zisi, V., Giannitsopoulou, E., Vassiliadou, O., Pollatou, E. and Kiomourtzoglou, E. (2009) Performance level, abilities and psychological characteristics in 
Rev.int.med.cienc.act.fís.deporte - vol. 18 - número 72 - ISSN: 1577-0354

young junior rhythmic gymnasts: The role of sport experience.

International Quarterly of Sport Science, 1(4), 1-13.

Número de citas totales / Total references: 35 (100\%)

Número de citas propias de la revista / Journal's own references: 2 (5,71\%)

Rev.int.med.cienc.act.fís.deporte - vol. 18 - número 72 - ISSN: 1577-0354 